\title{
Discurso de aceptación del Premio Biología Tropical 2014
}

Entregado el 19 de setiembre del 2014, en la Escuela de Biología de la Universidad de Costa Rica, Ciudad Universitaria Rodrigo Facio, San Pedro de Montes Oca, San José, Costa Rica.

Christopher Vaughan

Estimada Dra. Alice Pérez Sánchez, Vice-Rectora de Investigación, Universidad de Costa Rica; Señor Catedrático Julián Monge, Director de la Revista de Biología Tropical; Señores integrantes del Comité Editorial de la Revista de Biología Tropical; Estimados colegas, ex-estudiantes (hoy día profesionales destacados);

Familiares queridos, amigos y madre naturaleza:

\section{"The world will not evolve past its current state of crisis by using the same thinking that created the situation."}

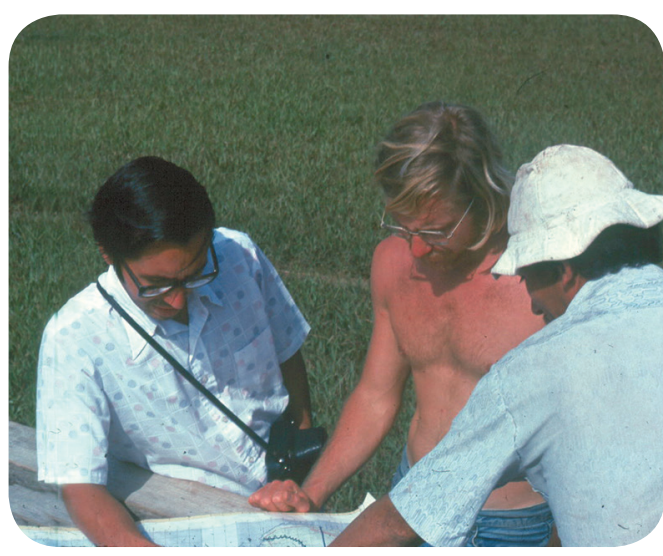

Mario Boza y Christopher Vaughan dentro del futuro Parque Nacional Corcovado discutiendo los límites finales con Panamá, y nuestro baqueano.

Agradezco profundamente a la Revista de Biología Tropical y a la Universidad de Costa Rica por hacerme entrega del Premio de Biología Tropical-2014. Es un honor recibir tan alta
Albert Einstein

distinción, otorgada por esta revista de difusión y renombre mundiales, la cual tiene además el mérito de publicarse ininterrumpidamente desde el año de 1953. Ha sido un enorme privilegio para mí, mis colegas, asistentes y estudiantes, haber podido publicar en ella el resultado de nuestros estudios, desde al menos el año de 1987.

Sin embargo, este honor no es exclusivamente mío. Es mi deseo hacer extensivo este reconocimiento también a mis colegas, tanto a los que hoy nos acompañan, como a aquellos que no están presentes en esta aula, pero con quienes he estudiado, trabajado y luchado por comprender y conservar la vida silvestre a lo largo de casi medio siglo, en diferentes instituciones: desde la Escuela de Ciencias 


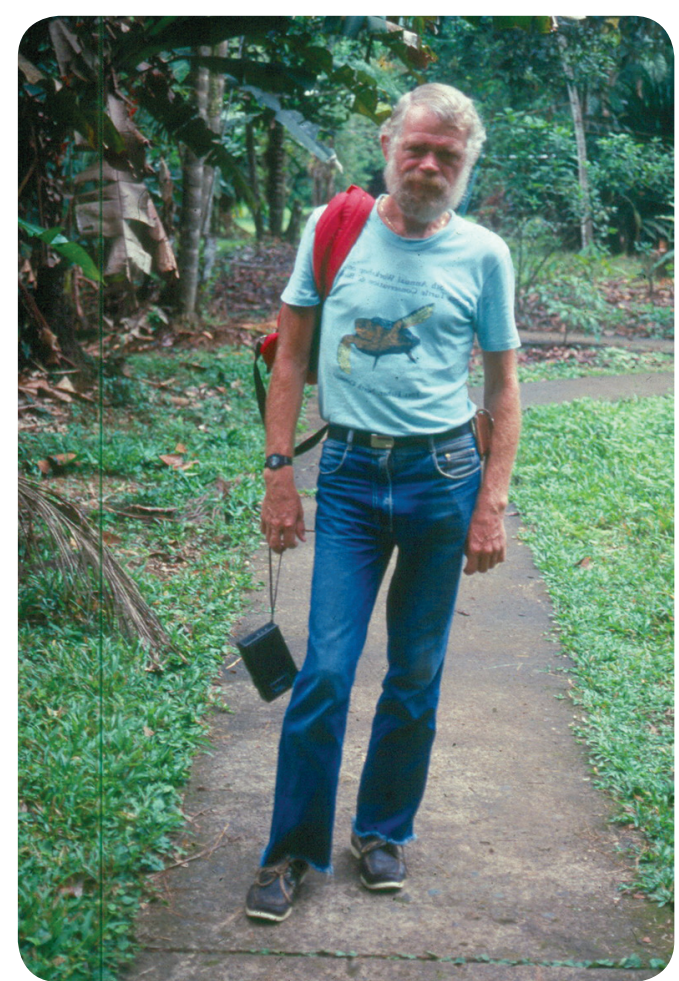

Douglas Robinson en una gira de su curso de Herpetología de la UCR.

Ambientales y el Programa en Manejo de Vida Silvestre de la Universidad Nacional hasta el Departamento de Parques Nacionales; desde el Departamento de Ecología Forestal y Vida Silvestre de la Universidad de Wisconsin-Madison y la A.C.M. (Asociación de Universidades del Medio Oeste) (USA) hasta, espero, esta Escuela de Biología de la Universidad de Costa Rica; desde las comunidades locales, hasta las instituciones estatales y las privadas.

Llegué a Costa Rica por tierra a los 19 años, en 1969, tras manejar dos semanas y media desde San Francisco. Bastante simbólico es el hecho de que el automóvil expiró nomás llegamos a territorio tico. Algo me decía que llegué para quedarme. Fui durante ese año estudiante de biología tropical en la A.C.M. Mi interés por comprender esta nueva y exuberante naturaleza, a la que no estaba habituado, me llevó a trabar amistad con quienes serían mis primeros mentores: Charles Schnell (con proyectos de investigación de campo y la literatura de biología tropical); el recordado Dr. Rafael Lucas Rodríguez Caballero, ex director de la Escuela de Biología de la Universidad de Costa Rica, quien bondadosamente identificaba y dibujaba las orquídeas que le traía, y respondía con generosidad a mis constantes preguntas, acompañado muchas veces por su amigo don Manuel Chavarría; Dr. Douglas Robinson me contestaba otras preguntas y el Dr. Dan Janzen, quien fuera mi director en un proyecto de investigación.

Apenas graduado de Grinnell College, regresé nuevamente a CR en 1971, como voluntario del Cuerpo de Paz, a colaborar con el recién creado Departamento de Parques Nacionales. Don Mario Boza, quien fungiera como director, me envió a trabajar en el Centro Científico Tropical con los doctores Leslie Holdridge, Alexander Skutch y Joseph Tosi, los cuales realizaban un inventario de potenciales parques nacionales y reservas biológicas. Tan sólo existían tres en ese entonces. Sus conocimientos, humildad y pasión hacia la naturaleza, fueron y continúan siendo mi inspiración. Mi tarea era ubicar sitios propicios para nuevos parques, o sea, recorrer todo el territorio nacional. Me entregaron una moto y me desearon suerte. Finalmente, en moto o en bote, a pie o

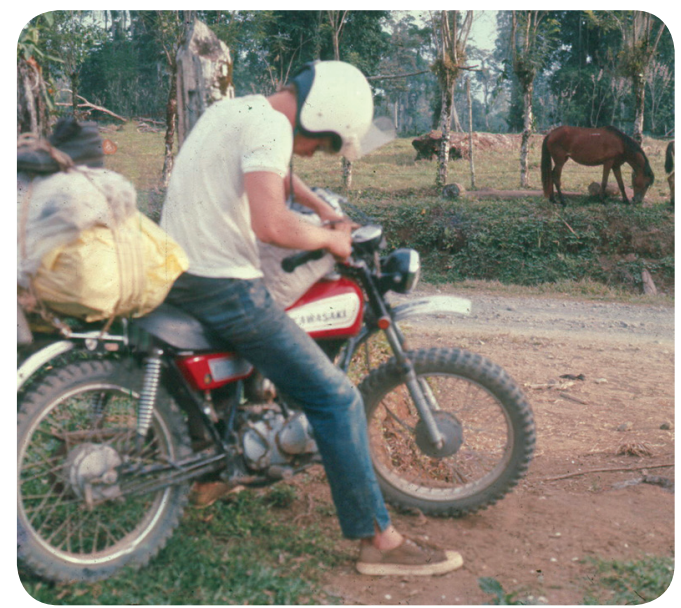

Christopher Vaughan en gira para identificar potenciales áreas para establecer parques y reservas. 

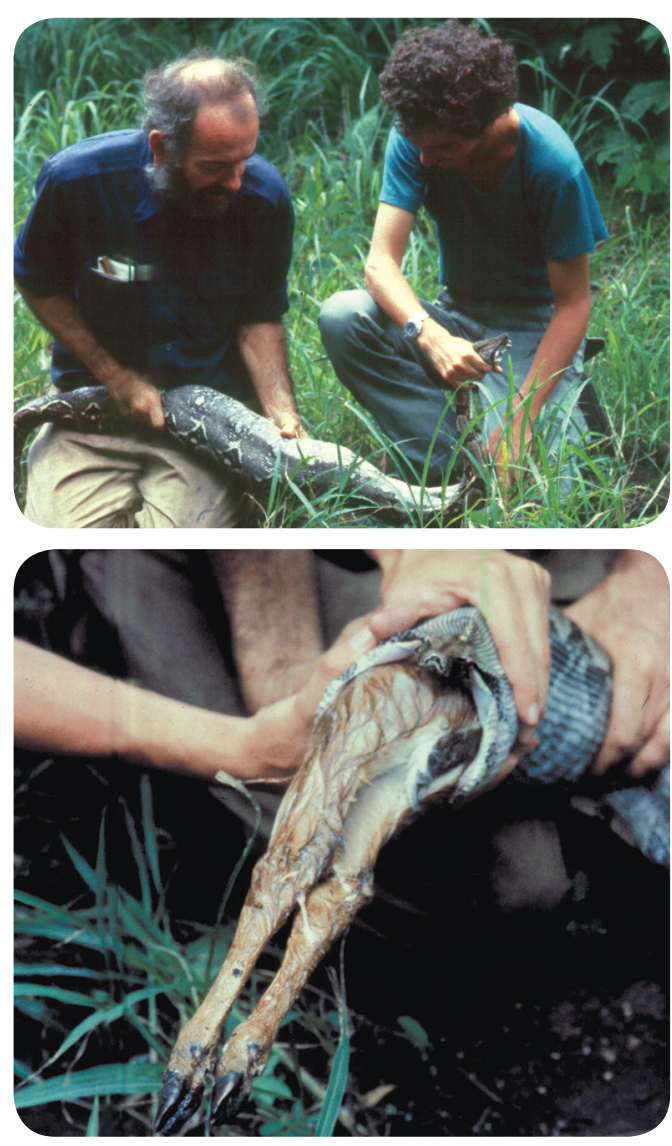

Daniel Janzen y Eduardo Carrillo (estudiante de la Primera Promoción de la Maestría en Manejo de Vida Silvestre) sostienen una boa con un cervatillo.

en avioneta, solo o con Adelaida Chaverri, Luis Poveda, y a veces Gary Stiles y Julio Sánchez de la Escuela de Biología, exploramos el país desde Osa hasta la Barra del Colorado, desde la Isla Bolaños hasta Gandoca, Manzanillo, la casi totalidad de Guanacaste, y cruzamos la Cordillera de Talamanca dos veces. El problema es que mis andanzas en el campo lo que hicieron fue enrumbar más mi vida hacia el estudio y la investigación. Cuando me percaté, además de ubicar lo que luego serían nuestros parques de Chirripó, Caño Negro, Corcovado o Manuel Antonio, tomaba también anotaciones de cuanto veía. Descubrí heces de grandes felinos llenas de espinas y constaté que los puercoespines constituían uno de sus platillos. Don
"Glance at the sun,

See the moon and the stars, Gaze at the beauty of earth's greenings, Now, think."

Hildegard Von Bingen

Luis Diego Gómez escuchaba mis descubrimientos y fue el primero que, con sabiduría, me instó a publicar la nota que redacté al respecto. Esta, mi primera publicación, vería gracias a él la luz en Brenesia. Dan Janzen resumió luego su consejo en una máxima que he hecho mía, y que inculco en mis alumnos: "Si no se publica, no es investigación."

Publiqué, entonces, como me enseñaron mis maestros. Entre ellos, el Dr. Luis Fournier Origgi, quien dirigió mi tesis de maestría, un plan de manejo del Parque Nacional Corcovado. Ya en 1975 empecé a laborar en la UNA, en Ciencias Ambientales, donde permanecí durante 28 años, introduciendo investigaciones y creando un énfasis en los estudios, hacia la vida silvestre. En 1982 creamos una Licenciatura en Manejo de Vida Silvestre, la cual al poco tiempo se convirtió en la primera Maestría de dicha especialidad en toda América Latina. Cumple actualmente 30 años y ha graduado al día de hoy 180 estudiantes de 24 países. Se han

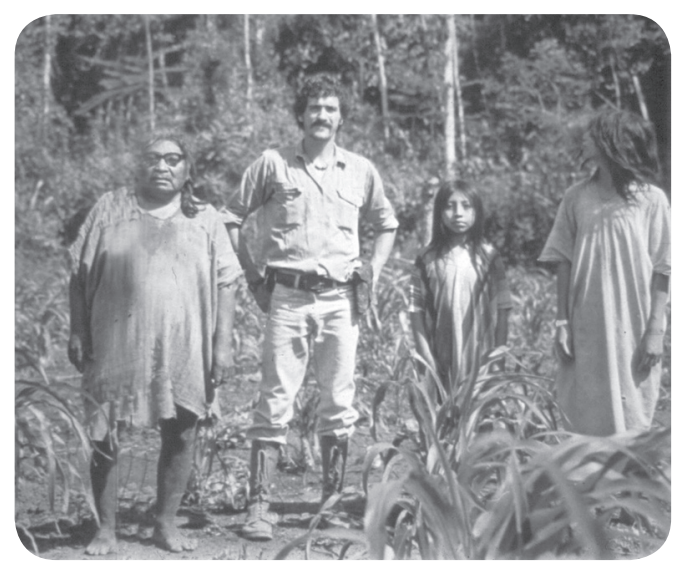

Ignacio March, estudiante de la Primera Promoción de la Maestría en Manejo de Vida Silvestre, documentando la utilización de la vida silvestre en los indígenas de la Selva Lacandona, México. 
llevado a cabo múltiples proyectos de investigación de tesis por parte de estudiantes en muchos países tropicales, en los cuales posteriormente se han constituido como líderes pioneros y han alcanzado puestos clave. El manejo de la vida silvestre no implica simplemente su estudio. El manejo de la vida silvestre implica lidiar con funcionarios, convencer políticos, mover comunidades, involucrar al sector privado, y además, adaptarse a imprevistos sobre la marcha. La maestría proveía a los alumnos de las herramientas necesarias para hacerlo, a través de proyectos en los que se entrecruzaban los aspectos ecológicos con los socioeconómicos e institucionales. Entre los proyectos modelo llevados a cabo (los cuales combinaban investigación, docencia y extensión) se destacan: "Ecología y manejo de humedales en Palo Verde", "Ecología y manejo de la lapa roja en el Pacifico Central", así como "Ecología y manejo del venado colablanca en la Isla San Lucas" (donde tuve ocasión de ganarle varias partidas de ajedrez a uno de los llamados "Hijos del Diablo", y de perder estratégicamente la última, el día de mi partida).

Además, entre 1975 y 1997, queriendo imitar el estilo de vida de Alexander Skutch en su propiedad El Quizarrá y "regresar a la tierra” como algunos "hippies" de la época, mi familia y yo vivimos en fincas al norte de Heredia. En la primera construimos una casa con madera mayoritariamente de la finca, que por una década funciono únicamente a base de energía solar, gas propano, candelas y bombillas alimentadas por baterías. Muchos de nuestros alimentos, los

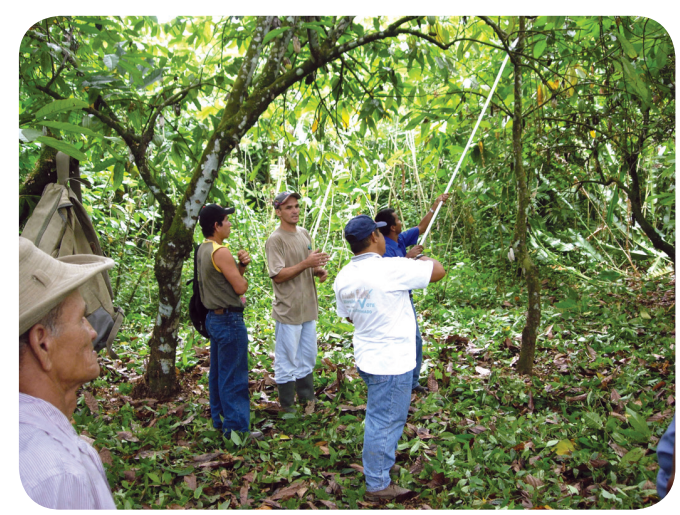

Se puede manejar los cacaotales a través de la poda para asegurar la producción de cacao y su biodiversidad.

cuales incluso vendimos o donamos, provenían de nuestra lechería, aves de corral, colmenas, huerto o árboles frutales.

En el año 1997, mi familia y yo fuimos para el norte, y tuve luego oportunidad de doctorarme en el Instituto de Estudios Ambientales Gaylord Nelson de la Universidad de Wisconsin-Madison y luego ejercer la docencia entre ese instituto y el Departamento de Vida Silvestre allí, el primero en haber sido fundado en el mundo. Durante ese período mi pasión y mi nostalgia me trajeron unas cuatro veces por año a Costa Rica, a impartir cursos breves en la Maestría de Vida Silvestre en la UNA, donde transmitía los conocimientos adquiridos, y a continuar con mis investigaciones sobre la lapa roja. También iniciamos estudios sobre el cacao, a fin de investigar la producción y biodiversidad de las pequeñas plantaciones,

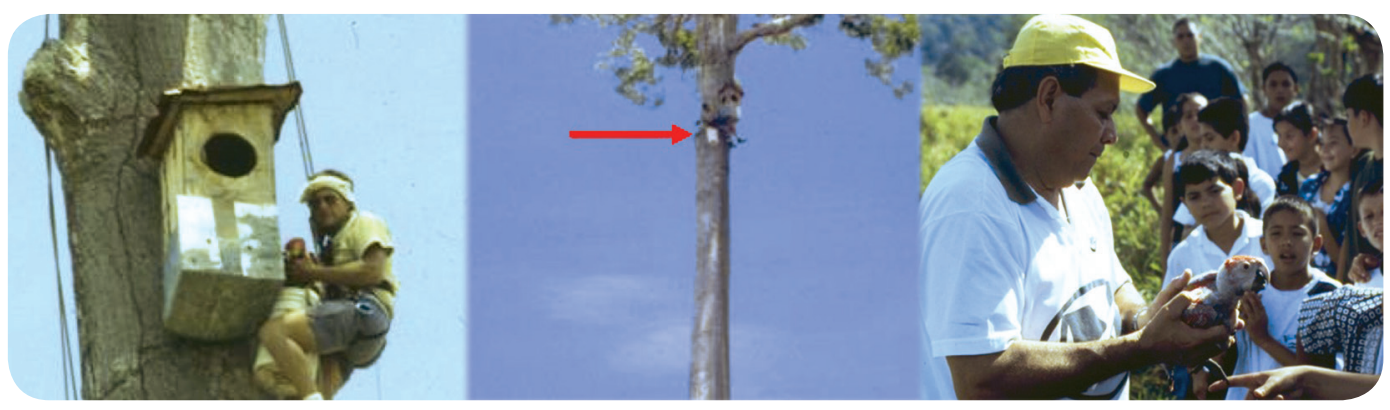

Nidos artificiales para lapa (Ara macao) como herramientas de investigación, manejo, y extensión en las comunidades aledañas. 


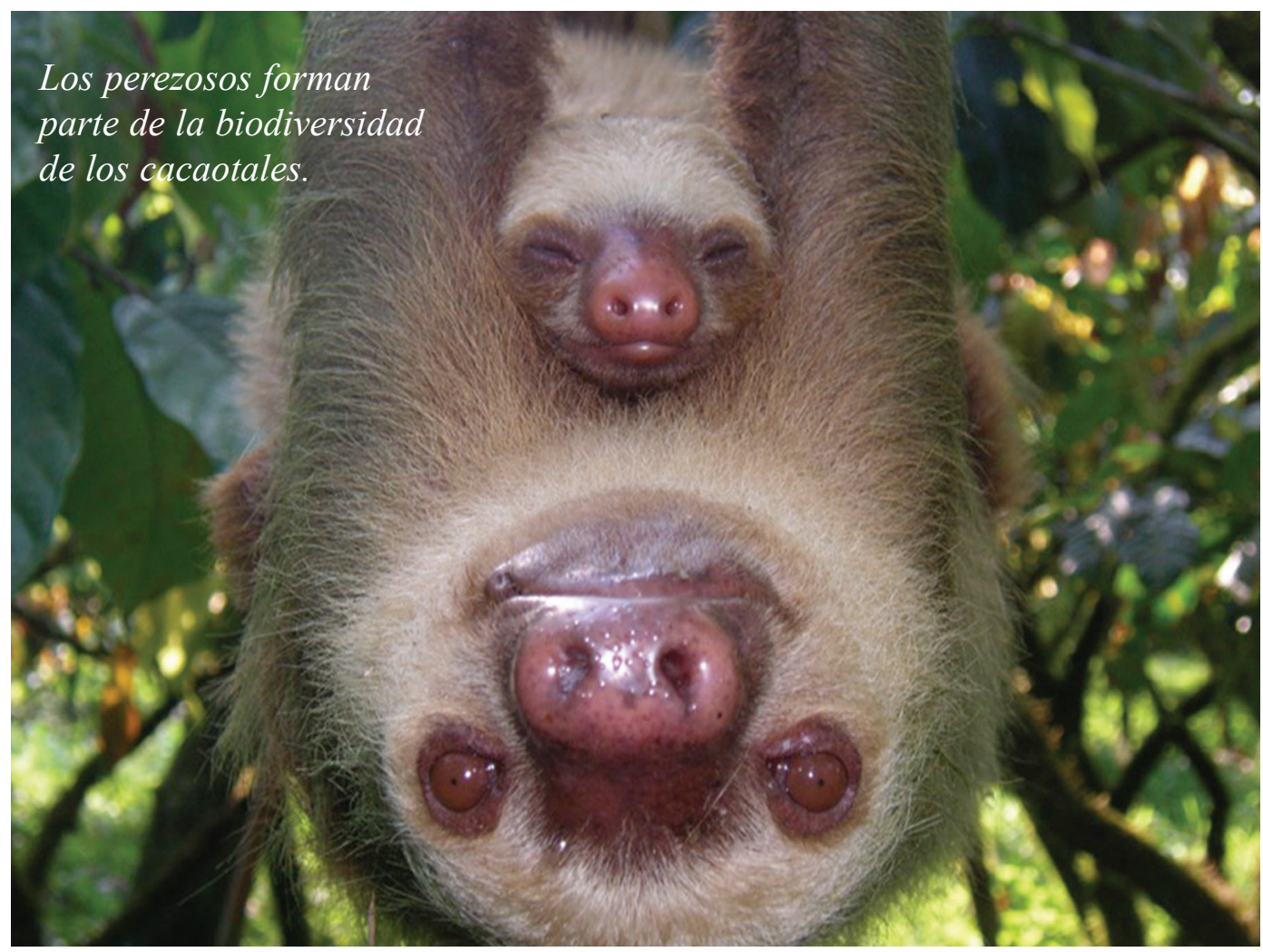

enfocando los estudios de biodiversidad particularmente en arañas y perezosos.

Es en el año 2009 cuando, designado como director de la A.C.M., retorno definitivamente al país, a consolidar -con la ayuda inestimable de asesores altamente calificados-, un sueño largamente acariciado por mí: propiciar que los estudiantes de pregrado no sólo se restrinjan a un estudio académico, sino capacitarlos para llevar a cabo una investigación científica, cuyos datos y resultados, redactados en forma técnica, se conviertan también en una herramienta útil para incidir en la sociedad, mejorar la realidad que les circunda y a la vez empoderarlos como personas. Mi agradecimiento reiterado a la Revista de Biología Tropical por la publicación de treinta y seis de dichos trabajos, nueve de los cuales aparecen en su más reciente edición.

No quiero finalizar sin agradecer al Dr. Gustavo Gutiérrez, Director de la Escuela que hoy nos recibe, por el generoso gesto de invitarme, en este punto de mi carrera, a continuar mi tarea en el seno de esta institución. Espero poder contribuir en algo a la valiosa labor de mis colegas y a la evolución de esta escuela, pieza fundamental en el estudio y conservación de nuestra biodiversidad.

Finalmente, deseo ofrecer este reconocimiento de que se me hace objeto, junto con mi amor y mi devoción, al verdadero motivo que nos convoca hoy, y al que hemos dedicado el esfuerzo de toda una existencia: la naturaleza, la cual, a pesar de la fragilidad de su equilibro y de encontrarse severamente amenazada, hasta el presente ha hallado siempre la resiliencia necesaria para resistir, y recordarnos, con su imponencia, nuestra pequeñez y nuestra humildad. A ella, mi homenaje, mi respeto y mi inclaudicable compromiso de protegerla y comprenderla, y de guiar a mis estudiantes a hacer lo mismo. A TODOS UDS., MI AMISTAD.

Namaste 
